\title{
The New Normal? Patient Satisfaction and Usability of Telemedicine in Breast Cancer Care
}

\author{
Bryan A. Johnson, BS ${ }^{1}$, Bruce R. Lindgren, $\mathrm{MS}^{2}$, Anne H. Blaes, $\mathrm{MD}^{3}$, Helen M. Parsons, PhD, MPH ${ }^{4}$, \\ Christopher J. LaRocca, MD ${ }^{5}$, Ronda Farah, $\mathrm{MD}^{6}$, and Jane Yuet Ching Hui, MD, $\mathrm{MS}^{2,5}{ }_{\mathbb{D}}$ \\ ${ }^{1}$ University of Minnesota Medical School, Minneapolis, MN; ${ }^{2}$ Masonic Cancer Center, University of Minnesota, \\ Minneapolis, MN; ${ }^{3}$ Division of Hematology, Oncology and Transplantation, Department of Medicine, University of \\ Minnesota, Minneapolis, MN; ${ }^{4}$ Division of Health Policy and Management, University of Minnesota School of Public \\ Health, Minneapolis, MN; ${ }^{5}$ Division of Surgical Oncology, Department of Surgery, University of Minnesota, Minneapolis, \\ MN; ${ }^{6}$ Department of Dermatology, University of Minnesota, Minneapolis, MN
}

\begin{abstract}
Background. Telemedicine was adopted to minimize exposure risks for patients and staff during the coronavirus disease 2019 pandemic. This study measured patient satisfaction and telemedicine usability in breast cancer care.

Methods. Adult breast cancer patients who had a telemedicine visit at a single academic institution (with surgical, radiation, or medical oncology) from 15 June 2020 to 4 September 2020 were surveyed anonymously. Patient and cancer characteristics were collected, and patient satisfaction and telemedicine usability were assessed using a modified Telehealth Usability Questionnaire with a 7-point Likert scale. Associations of satisfaction and usability with patient characteristics were analyzed using Wilcoxon rank-sum and Kruskal-Wallis tests.
\end{abstract}

Results. Of 203 patients who agreed to be contacted, 78 responded, yielding a response rate of $38 \%$. The median age of the respondents was 63 years (range 25-83 years). The majority lived in an urban area $(61 \%)$, were white $(92 \%)$, and saw a medical oncologist $(62 \%)$. The median patient satisfaction score was 5.5 (interquartile range [IQR] 4.25-6.25). The median telemedicine usability score was 5.6 (IQR 4.4-6.2). A strong positive correlation was seen

(C) Society of Surgical Oncology 2021

First Received: 22 April 2021

Accepted: 2 June 2021;

Published Online: 17 July 2021

J. Y. C. Hui, MD, MS

e-mail: jhui@umn.edu between satisfaction and usability, with a Spearman correlation coefficient $(\rho)$ of $0.80(p<0.001)$. Satisfaction and usability scores did not vary significantly according to patient age, race, location of residence, insurance status, previous visit commute time, oncology specialty seen, prior telemedicine visits, or whether patients were actively receiving cancer treatment.

Conclusions. Breast cancer patients were satisfied with telemedicine and found it usable. Patient satisfaction and telemedicine usability should not limit the use of telemedicine in future post-pandemic breast cancer care.

Coronavirus disease-2019 (COVID-19) has prompted health systems to rethink how health care can be delivered in a safe and effective way. To mitigate the risk of COVID19 transmission between patients, physicians, and nonphysician providers, many health systems have rapidly expanded their use of telemedicine. ${ }^{1-3}$ Policy changes have further supported this transition in care delivery, namely, relaxation of technology requirements for virtual communication platforms, temporary expansion of recommended telemedicine use cases, and ability of health systems to bill telemedicine services as if they were performed in person. ${ }^{4,5}$ Accompanying this temporary expansion in virtual care has been a surge in the literature critically assessing the long-term utility of telemedicine within various care disciplines and patient populations. ${ }^{6-11}$

The use of telemedicine in oncologic care is of particular interest for two primary reasons. First, patients with cancer often are immunocompromised from their cancer or the treatments they receive, such as chemotherapy or 
corticosteroids. ${ }^{12}$ Second, patients with cancer, by the nature of their care, have a high number of interactions with the health care system, increasing their risk of exposure. ${ }^{13}$ Data suggest that patients with cancer may be at increased risk of SARS-CoV-2 infection and severe manifestations of COVID-19. ${ }^{14-16}$ However, consideration of telemedicine as a long-term option in oncologic care extends beyond infection risk reduction. For a population in which $35-45 \%$ of all patients with cancer experience psychological distress secondary to their cancer diagnosis and course of care at baseline, additional distress caused by a new approach to care delivery may be unfavorable. ${ }^{17-19}$ Thus, assessment of patient-centered metrics such as satisfaction and usability is of high importance.

Given the growing prevalence of telemedicine secondary to the COVID-19 pandemic and the importance of telemedicine in oncologic care, our study aimed to assess patient satisfaction and usability of telemedicine in the breast cancer patient population to determine the role of telemedicine in future post-pandemic breast cancer care.

\section{METHODS}

A cross-sectional study of adult ambulatory patients with breast cancer was conducted using an anonymous survey to assess patient satisfaction and usability of telemedicine. The study participants were patients from a single academic institution in Minnesota. Eligible patients were informed of the survey study by their physician at the conclusion of their telemedicine appointment and given the opportunity to participate in the study. The patients opting to participate were sent a link to an electronic informed consent form and survey by email using the Research Electronic Data Capture (REDCap) system through the University of Minnesota within 2 days after their appointment. ${ }^{20}$ If a participant did not complete the survey after the first email, no secondary attempts were made to prompt survey completion. Data collection occurred in a 12-week period during the COVID-19 pandemic, from 15 June 2020 to 4 September 2020.

\section{Inclusion and Exclusion Criteria}

The study enrolled adult English-speaking patients with a former or current breast cancer diagnosis who currently were using telemedicine for health care visits with a surgical oncologist, medical oncologist, and/or radiation oncologist consenting to participate in the survey study. Patients who had telemedicine appointments with physicians of different oncologic subspecialties during the study period were eligible to complete the survey multiple times.

\section{Study End Points and Survey}

The primary end point of the study was patient satisfaction with telemedicine, and the secondary end point was the usability of telemedicine. Both end points were assessed independently and in relation to patient demographic data (age, sex, race/ethnicity, urban vs urban cluster vs rural residence), health care coverage, telemedicine visit characteristics (first or subsequent visit with a physician, presence of an active health concern during the visit, presence of connectivity issues during the visit, subspecialty of the physician, telemedicine appointment frequency and type, technological barrier to first-time use of telemedicine), cancer and treatment characteristics (time since diagnosis, stage, completed/current/planned therapies), and prior cancer care delivery statistics (in-person appointment frequency and commute time).

We created an anonymous survey consisting of 40 questions divided into four sections: demographics (6 questions), visit history (11 questions), cancer history and treatment (6 questions), and telemedicine experience (17 questions) (see Supplementary File for the survey questions). The Telemedicine Experience section contained questions adapted from the Telehealth Usability Questionnaire (TUQ), a validated survey tool developed and designed by Parmanto et al. ${ }^{21}$ to assess the primary and secondary end points. The survey items in the Telemedicine Experience section used a 7-point Likert scale ranging from 1 (strongly disagree) to 7 (strongly agree).

The participants selected their level of agreement with provided statements on usefulness, ease of use and learnability, interface quality, interaction quality, reliability, satisfaction, and future use. The primary end point was based on an overall score from the "reliability" and "satisfaction and future use" subcategories of our survey, and the secondary end point was based on an overall score from the "usefulness," "ease of use and learnability," "interface quality," and "interaction quality" subcategories of our survey.

\section{Statistical Analysis}

For each participant, a composite score representing the mean numeric score from the 7-point scale of the patient satisfaction (primary end point) survey items was calculated. The composite scores across multiple participants were summarized using the median and interquartile range (IQR). The same process was repeated for the telemedicine usability (secondary end point) survey items.

The statistical analysis used non-parametric methods to relate these scores to patient, visit, cancer, and treatment 
factors. Comparisons between two or more groups were performed using the Wilcoxon rank-sum test or the Kruskal-Wallis test. The Spearman rank correlation coefficient was used to assess the association between the primary and secondary end points.

All $p$ values lower than or equal to 0.05 were considered statistically significant. The statistical analysis was performed using SAS (version 9.4) software. The study protocol was reviewed and approved by the University of Minnesota Institutional Review Board. All the survey participants provided written consent before survey participation. They could exit and close the survey at any time, although the responses already provided were included in the analysis.

\section{RESULTS}

Of 783 eligible patients, 310 were screened for participation. Of these 310 patients, 203 opted in to receive the survey, and 78 patients responded, yielding a response rate of $38.4 \%$. The survey was completed by 75 respondents, and they were included in the final analysis (Fig. 1).

The survey participants had a median age of 63 years. The participants were predominately white $(91.9 \%)$, resided in an urban area (61.3\%), and were insured privately and/or through Medicare (84.7\%). Most of the participants had breast cancer diagnosed more than 12 months before their appointment $(60.8 \%)$, and more than half of the participants (56.7\%) had already completed all elements of therapy (Table 1). Regarding specific treatments, most of the participants had already undergone surgical resection

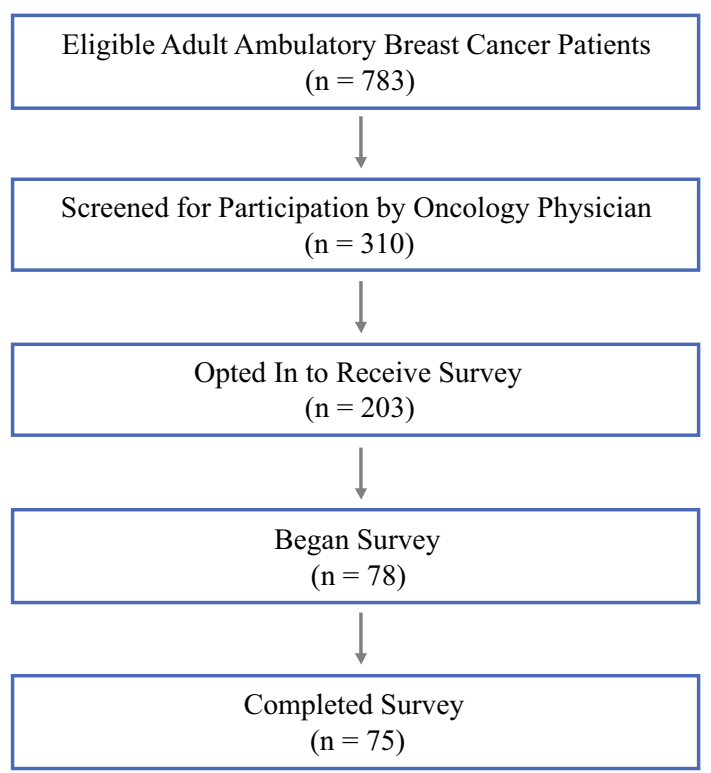

FIG. 1 Flowchart of breast cancer patients in the patient satisfaction and telemedicine usability survey
(85.3\%) and had completed radiation therapy (62.7\%), with many also having completed or still receiving chemotherapy $(49.4 \%)$.

The prior experience of the participants with telemedicine was minimal. Most had attended fewer than two telemedicine visits in the past $(58.1 \%)$. During the telemedicine visit, most of the patients did not report any connection trouble $(61.3 \%)$. The majority of the patients had a specific health issue to discuss $(56 \%)$, were seen at a return visit $(93.3 \%)$, and had a visit with a medical oncologist $(62.7 \%)$ (Table 2).

\section{Patient Satisfaction with Telemedicine}

The median patient satisfaction scores were 5.5 (IQR 4.25-6.25) overall and 4.75 (IQR 3.75-5.75) for the respondents who saw a surgical oncologist. The patient

TABLE 1 Demographics, cancer, and treatment characteristics of the survey respondents $(n=75)$

\begin{tabular}{ll}
\hline Characteristic & $n(\%)$ \\
\hline Median age: years (range) & $63(25-83)$ \\
Race & \\
White & $68(91.9)$ \\
Black & 0 \\
Asian & $3(4.1)$ \\
Other & $3(4.1)$ \\
Residence (by population size) & \\
Urban: $>50,000$ & $46(61.3)$ \\
Urban cluster: 2500-50,000 & $23(30.7)$ \\
Rural: $<2500$ & $6(8.0)$ \\
Medical insurance & \\
Private health insurance & $33(45.8)$ \\
Medicare & $10(13.9)$ \\
Private and Medicare or Medigap & $25(34.7)$ \\
Other & $4(5.6)$ \\
Date of breast cancer diagnosis & \\
$<6$ months ago & $18(24.3)$ \\
6-12 months ago & $11(14.9)$ \\
$>12$ months ago & $45(60.8)$ \\
Presence of stage IV disease & \\
Yes & $11(34.4)$ \\
No & $21(65.6)$ \\
Treatment completion & \\
I am still receiving treatment & $30(40.5)$ \\
Completed $<12$ months ago & $18(24.3)$ \\
Completed $>12$ months ago & $23(32.4)$ \\
\hline The & \\
\hline
\end{tabular}

The sum of the responses for each characteristic may be $<75$ due to missing survey data. 
TABLE 2 Visit characteristics of the survey respondents $(n=75)$

\begin{tabular}{ll}
\hline Characteristic & $n(\%)$ \\
\hline Oncology subspecialty & \\
Surgical & $16(21.3)$ \\
Medical & $47(62.7)$ \\
Radiation & $12(16.0)$ \\
Care history with oncologist & \\
New (first visit) & $5(6.7)$ \\
Established (subsequent visit) & $70(93.3)$ \\
Presence of new or ongoing problem to discuss at visit & \\
Yes & $42(56.0)$ \\
No & $33(44.0)$ \\
Type of visit(s) in the past 4 weeks & \\
Video only & $28(37.8)$ \\
Phone only & $28(37.8)$ \\
Video and phone only & $7(9.5)$ \\
Other, including in-person & $11(14.9)$ \\
No. of telemedicine visits attended & \\
1 & $22(29.7)$ \\
2 & \\
\hline & \\
\hline &
\end{tabular}

The sum of the responses for each characteristic may be $<75$ due to missing survey data.

satisfaction score demonstrated a strong positive correlation with usability (Spearman correlation coefficient $[\rho]$, $0.80 ; p<0.001)$. Patient characteristics, including location of residence $(p=0.421)$ and type of insurance coverage $(p=0.706)$, were not significantly related to satisfaction scores (Table 3). Additionally, the patient satisfaction scores were not significantly associated with treatment type (breast cancer surgery $[p=0.078]$, chemotherapy [ $p=0.962]$, radiation [ $p=0.077]$, and endocrine therapy $[p=0.181])$ or whether the treatment was completed, ongoing, or upcoming (Fig. 2). Furthermore, the patient satisfaction scores also showed no association with visit characteristics such as oncologist subspecialty $(p=0.129)$, history of care with the oncologist $(p=0.492)$, and presence of a problem to discuss at the telemedicine visit $(p=0.808)$. However, the presence of connection trouble was significantly related to a change in patient satisfaction $(p=0.048)$. Specifically, patients who encountered connection issues and were able to resolve them to continue the visit expressed lower satisfaction (Table 4).

\section{Usability of Telemedicine}

The median telemedicine usability scores were 5.6 (IQR 4.4-6.2) overall and 5.4 (IQR 3.2-5.8) among respondents seeing a surgical oncologist. Differences in telemedicine usability scores were not associated with time since cancer diagnosis (Table 4), treatment type (breast cancer surgery [ $p=0.335]$, chemotherapy $[p=0.471]$, radiation therapy $[p=0.327]$, and endocrine therapy $[p=0.245])$ or completion of treatment (Fig. 3). Furthermore, usability of telemedicine scores also were not associated with visit characteristics, including the number of previous telemedicine visits attended $(p=0.834)$, the frequency of telemedicine visits $(p=0.268)$, and the need for patients to upgrade their phone, tablet, or computer in order to attend telemedicine visits $(p=0.130)$. However, the presence of connection trouble, either resolved or requiring a transition to a different form of visit, was associated with lower usability scores than the absence of connection trouble $(p=0.001 ;$ Table 4$)$.

\section{DISCUSSION}

The 1990s saw a surge in literature assessing patient satisfaction with telemedicine across many medical disciplines spanning psychiatry, dermatology, otolaryngology, hospice, and home nursing, among others. ${ }^{22}$ A limited number of studies then were performed to evaluate patient satisfaction with video-conference-based physician consultations exclusively in the setting of oncologic care. ${ }^{23}$ These studies generally reported that video-conferencing was favored by patients over in-person visits. However, small study sizes, no studies specific to breast cancer patients, and the absence of a scientifically validated satisfaction or usability survey to measure satisfaction outcomes highlight where such literature is lacking. Furthermore, some of these studies were conducted during a technologically different time in which patients required 
TABLE 3 Patient satisfaction and telemedicine usability scores compared with patient, cancer, and treatment characteristics $(n=75)$

\begin{tabular}{|c|c|c|c|c|}
\hline Characteristic & Median satisfaction score ${ }^{\mathrm{a}}$ (IQR) & $p$ value $^{\mathrm{b}}$ & Median usability score $^{\mathrm{a}}$ (IQR) & $p$ value $^{\mathrm{b}}$ \\
\hline Race & & 0.112 & & 0.065 \\
\hline White & $5.42(4.0-6.0)$ & & $5.6(4.4-6.1)$ & \\
\hline Black & N/A & & N/A & \\
\hline Asian & $6.25(6.0-6.5)$ & & $6.6(6.0-6.75)$ & \\
\hline Other & $6.0(5.25-7.0)$ & & $6.0(6.0-7.0)$ & \\
\hline Residence (by population size) & & 0.421 & & 0.500 \\
\hline Urban: $>50,000$ & $5.25(4.0-6.25)$ & & $5.6(4.4-6.2)$ & \\
\hline Urban cluster: $2500-50,000$ & $5.75(5.0-6.25)$ & & $5.8(5.4-6.6)$ & \\
\hline Rural: $<2500$ & $5.13(2.0-6.0)$ & & $5.4(3.2-5.8)$ & \\
\hline Medical insurance & & 0.706 & & 0.489 \\
\hline Private health insurance & $5.5(4.75-6.0)$ & & $5.8(5.0-6.0)$ & \\
\hline Medicare & $5.75(4.0-6.25)$ & & $5.6(5.0-6.0)$ & \\
\hline Private and Medicare or Medigap & $5.38(3.75-6.25)$ & & $5.4(4.0-6.6)$ & \\
\hline Other & $4.88(3.88-5.0)$ & & $5.1(3.9-5.4)$ & \\
\hline Date of breast cancer diagnosis & & 0.228 & & 0.081 \\
\hline$<6$ months ago & $5.63(4.0-6.0)$ & & $5.3(4.0-6.0)$ & \\
\hline $6-12$ months ago & $4.88(3.75-5.5)$ & & $5.0(4.4-5.8)$ & \\
\hline$>12$ months ago & $5.75(5.0-6.25)$ & & $5.8(5.4-6.6)$ & \\
\hline Presence of stage IV disease & & 0.628 & & 0.233 \\
\hline Yes & $5.88(5.25-6.25)$ & & $6.0(5.6-6.0)$ & \\
\hline No & $5.42(4.5-6.13)$ & & $5.3(4.4-6.0)$ & \\
\hline Treatment completion & & 0.728 & & 0.292 \\
\hline I have not yet started treatment & $6.25(5.5-7.0)$ & & $6.8(6.6-7.0)$ & \\
\hline I am still receiving treatment & $5.42(4.5-6.13)$ & & $5.4(4.4-6.0)$ & \\
\hline Completed $<12$ months ago & $5.75(3.75-6.0)$ & & $5.9(4.0-6.6)$ & \\
\hline Completed $>12$ months ago & $5.5(4.0-6.25)$ & & $5.6(5.0-6.2)$ & \\
\hline
\end{tabular}

$I Q R$ interquartile range

The sum of the responses for each characteristic may be $<75$ due to missing survey data.

${ }^{\mathrm{a}}$ The maximum score is 7

${ }^{\mathrm{b}}$ The $p$ value is based on the Wilcoxon rank-sum test or the Kruskal-Wallis test

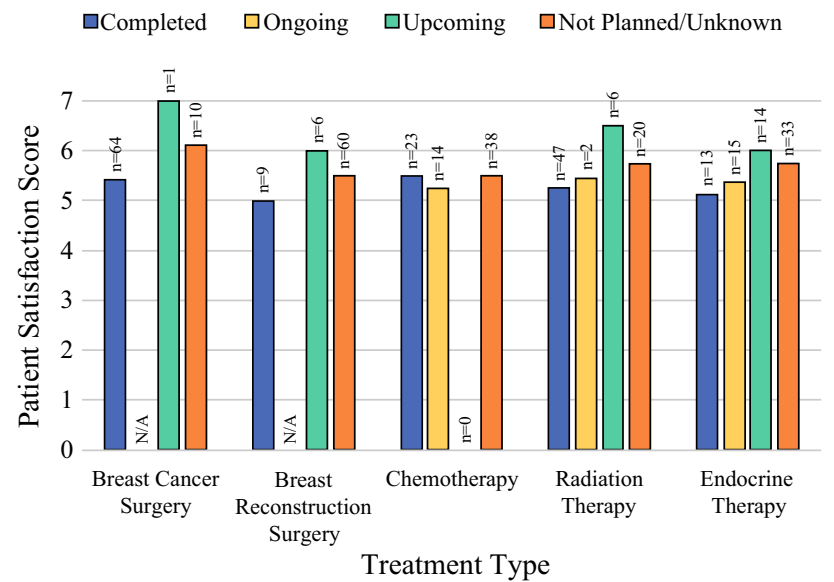

FIG. 2 Patient satisfaction scores on a 7-point Likert scale by status of breast cancer treatment $(n=75)$. For all treatment types, the $p$ value was higher than 0.05 hospital-provided video-capable hardware for video-conferencing to occur. ${ }^{24-26}$ The current era provides a stark contrast, in which telemedicine is software-based, with patients using their own devices.

Since the onset of the COVID-19 pandemic and the rapid shift to telemedicine by many health systems, literature assessing satisfaction of oncologic patients with telemedicine has expanded. However, recent studies have assessed patient satisfaction across all cancer types or within a specific oncologic subspecialty such as radiation oncology, and in many cases have done so without using a validated survey tool. ${ }^{27-30}$

Our study was unique in that it assessed satisfaction and usability specifically within the breast cancer population across all oncologic subspecialties using an adapted version of the TUQ, which consists of questions compiled 
TABLE 4 Patient satisfaction and telemedicine usability scores compared with visit characteristics $(n=75)$

\begin{tabular}{|c|c|c|c|c|}
\hline Characteristic & Median satisfaction score $^{\mathrm{a}}(\mathrm{IQR})$ & $p$ value ${ }^{\mathrm{b}}$ & Median usability score $^{\mathrm{a}}(\mathrm{IQR})$ & $p$ value $^{\mathrm{b}}$ \\
\hline Oncology subspecialty & & 0.129 & & 0.219 \\
\hline Surgical & $4.75(3.75-5.75)$ & & $5.4(3.2-5.8)$ & \\
\hline Medical & $5.75(5.0-6.25)$ & & $5.8(5.0-6.6)$ & \\
\hline Radiation & $5.75(5.0-6.13)$ & & $5.4(4.6-6.2)$ & \\
\hline Care history with oncologist & & 0.492 & & 0.063 \\
\hline New (first visit) & $5.33(5.0-5.5)$ & & $5.0(4.0-5.2)$ & \\
\hline Established (subsequent visit) & $5.5(4.25-6.25)$ & & $5.8(4.8-6.2)$ & \\
\hline Presence of new or ongoing problem to discuss at visit & & 0.808 & & 0.853 \\
\hline Yes & $5.5(4.25-6.25)$ & & $5.6(4.8-6.0)$ & \\
\hline No & $5.5(4.5-6.0)$ & & $5.6(4.2-6.6)$ & \\
\hline Type of visit(s) in the past 4 weeks & & 0.497 & & 0.622 \\
\hline Video only & $5.5(4.75-6.25)$ & & $5.8(5.0-6.6)$ & \\
\hline Phone only & $5.75(5.0-6.13)$ & & $5.9(4.7-6.4)$ & \\
\hline Video and phone only & $5.5(5.0-6.5)$ & & $5.2(4.4-5.8)$ & \\
\hline Other, including in-person & $4.75(3.5-5.75)$ & & $5.6(4.4-6.0)$ & \\
\hline No. of telemedicine visits attended & & 0.295 & & 0.834 \\
\hline 1 & $5.25(4.75-5.75)$ & & $5.6(5.0-6.0)$ & \\
\hline 2 & $5.63(3.88-6.25)$ & & $5.7(3.1-6.8)$ & \\
\hline$\geq 3$ & $5.75(4.75-6.25)$ & & $5.6(5.0-6.2)$ & \\
\hline Telemedicine visit frequency & & 0.317 & & 0.268 \\
\hline Less than once per month & $5.5(4.25-6.0)$ & & $5.6(4.4-6.0)$ & \\
\hline At least once per month & $5.88(4.75-6.25)$ & & $5.9(5.2-7.0)$ & \\
\hline Presence of telemedicine connection trouble & & 0.048 & & 0.001 \\
\hline Yes, could continue the visit & $4.88(3.88-5.88)$ & & $4.6(3.3-5.6)$ & \\
\hline Yes, had to reschedule the visit & $6.67(6.67-6.67)$ & & $5.2(5.2-5.2)$ & \\
\hline No & $5.75(5.0-6.25)$ & & $6.0(5.4-6.6)$ & \\
\hline Prerequisite upgrade of phone, tablet, or computer & & 0.121 & & 0.130 \\
\hline Yes & $4.25(2.63-5.5)$ & & $3.5(2.6-5.58)$ & \\
\hline No & $5.5(3.5-6.0)$ & & $5.6(5.0-6.2)$ & \\
\hline Most recent in-person appointment & & 0.471 & & 0.808 \\
\hline$<3$ months ago & $5.75(4.75-6.25)$ & & $5.6(4.8-6.2)$ & \\
\hline $3-6$ months ago & $5.0(3.5-6.0)$ & & $5.5(4.4-6.0)$ & \\
\hline$>6$ months ago & $5.5(5.0-6.0)$ & & $5.8(5.0-6.2)$ & \\
\hline In-person appointment commute time (min) & & 0.209 & & 0.326 \\
\hline $0-20$ & $5.0(4.13-5.63)$ & & $5.5(4.4-5.9)$ & \\
\hline $21-60$ & $5.75(4.88-6.25)$ & & $5.8(4.9-6.6)$ & \\
\hline$>60$ & $5.75(4.0-6.25)$ & & $5.8(5.2-5.8)$ & \\
\hline
\end{tabular}

The sum of the responses for each characteristic may be $<75$ due to missing survey data

$I Q R$ interquartile range

${ }^{\text {a }}$ The maximum score is 7

${ }^{\mathrm{b}}$ The $p$ value is based on the Wilcoxon rank-sum test or the Kruskal-Wallis test. The bold values denote statistical significance $(p<0.05)$

from various existing survey tools with proven content validity. ${ }^{31-33}$ All the TUQ survey subsections demonstrated "good" or "excellent" reliability as measured by Cronbach's coefficient alpha. ${ }^{21}$
In general, our study demonstrated agreement by breast cancer patients that they are satisfied with telemedicine and find it usable, with their perceptions of satisfaction tracking similarly with their perceptions of telemedicine usability. The absence of an association between satisfaction/ 


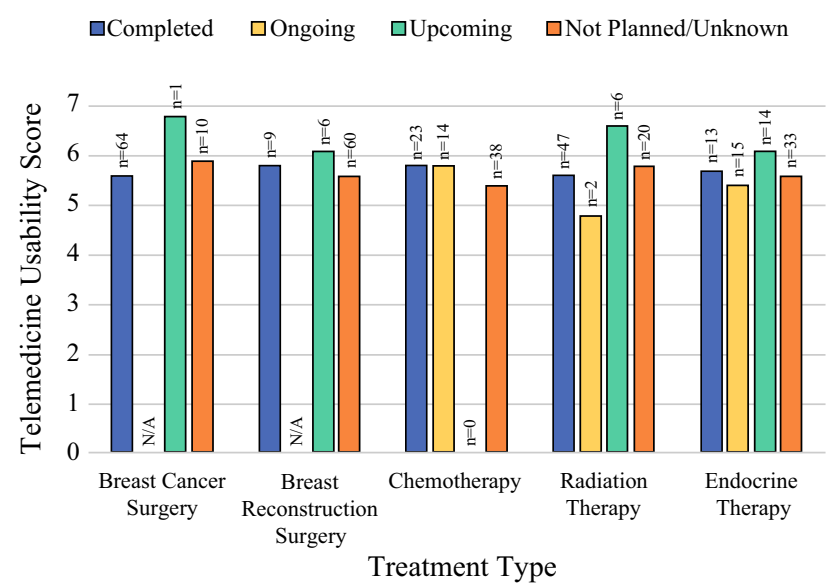

FIG. 3 Telemedicine usability scores on a 7-point Likert scale by status of breast cancer treatment $(n=75)$. For all treatment types, the $p$ value was higher than 0.05

usability with various demographic, visit, and cancer characteristics suggests the possibility that satisfaction and usability of telemedicine are both relatively stable within varying circumstances. In particular, the majority of the survey respondents were well-established breast cancer patients who either were actively receiving cancer treatments or had already completed treatment. Thus, these survey results may be most applicable in the follow-up visit setting.

We also did not observe differences in the patient satisfaction or telemedicine usability scores of the patients who had a new or ongoing problem in particular to address at the visit. This suggests that established breast cancer patients found telemedicine to be an acceptable mode of communication with their oncology physician, even when an active issue was present. Although we did not evaluate this aspect of telemedicine specifically, our electronic health record had a feature that allowed patients to send in electronic photos of their concerns (e.g., postoperative wound), allowing the physician or surgeon to remotely assess the concern or ask the patient to come in for a physical examination. Future work should include evaluation of this additional technology to enrich the characterization of telemedicine use.

When patients experienced connection trouble during their telemedicine visit, patient satisfaction and telemedicine usability scores were lower, indicating that technical difficulty may be a significant factor contributing to poorer patient perceptions of telemedicine satisfaction and usability. However, the need for technological devices (i.e., phone, tablet, or computer) did not seem to correlate with patient satisfaction or telemedicine usability, at least among the survey respondents who likely were inherently more "savvy" technologically because they had to respond to an electronic survey invitation and were willing to complete the survey online.

This survey study had several limitations. The study was conducted during the COVID-19 pandemic when in-person visits largely had ceased. Thus, we did not have the opportunity to compare the survey results with a control group of in-person visits. Relatedly, our data represents patient satisfaction and usability of telemedicine at one point in time within the context of a pandemic, and therefore may not be representative of satisfaction and usability in non-pandemic conditions. Our study was conducted a few months after the onset of the pandemic and after the adoption of telemedicine, which hopefully minimizes some of the technological difficulties inherent in navigating a brand new system, making the results more applicable to a post-pandemic world. Because telemedicine likely will continue to be a part of health care delivery, even after the pandemic, future work should include a comparison between telemedicine and in-person visits.

The study was anonymized to improve respondent recruitment, but due to the anonymous nature of the survey, no second attempt for a survey invitation could be sent if the patient did not respond, nor could a follow-up survey be completed at a later time during the COVID-19 pandemic. Furthermore, because a given patient could be invited to participate in the survey through different subspecialities, some individuals may have completed the survey more than once. Due to the anonymity of the survey, it was not possible to detect or account for these individuals.

Study size also was a limitation. We observed a high attrition rate, with only 75 respondents completing the survey despite distribution of the survey to 203 of the 310 initially screened patients who had agreed to be contacted about the study.

The study participants represented a small sample of patients with breast cancer of limited diversity. The respondents were predominately white and from one geographic area within a single health system. Non-Englishspeaking patients were excluded, and by default, those without Internet access or possession of electronic devices capable of connecting to a telemedicine visit also were excluded. Thus, the study design itself likely introduced a selection bias of breast cancer patients who were inherently more likely to be satisfied with telemedicine and to find telemedicine usable. Furthermore, the effect of disparities among this sample of the breast cancer patient population is unknown. The absence of baseline data defining usage of telemedicine by various demographic groups introduced ambiguity surrounding whether the selection bias in our 
study was founded on inequity in telemedicine access, inequity in access to an online survey, reluctance to complete a survey, or any combination thereof.

Our study measured telemedicine as either a video or a phone visit, and it did not examine the primary and secondary end points relative to visit, cancer, or treatment characteristics (i.e., postoperative visit vs preoperative visit, time since cancer diagnosis) within each oncologic subspecialty. This leaves the opportunity for future work to delineate the potential relationship of various telemedicine methods to patient satisfaction and usability. It also sets the stage for future work to explore how visit and treatment characteristics influence patients' perceptions of telemedicine across oncologic subspecialties in breast cancer care.

Finally, we recognize that oncology patients, particularly those with a new diagnosis, have unique psychosocial needs. We did not specifically evaluate patients' perceptions of telemedicine in this context. Additional study that includes anxiety and depression screening of in-person and telemedicine cohorts is needed to determine the usability of telemedicine in addressing the unique psychosocial needs of established and new oncology patients.

Despite the aforementioned limitations, the current study captured the adoption of telemedicine from the breast cancer patient perspective. Looking to the future, health care systems need to continue adjusting and normalizing operations to ensure the safety and well-being of patients, physicians, and non-physician providers while also prioritizing care delivery options that meet the needs of patients. In this light, we believe our study provides valuable context to such discussions, specifically within the realm of breast cancer care, bringing into focus what matters most in characterizing the role of telemedicine in health care moving forward.

Supplementary Information The online version contains supplementary material available at https://doi.org/10.1245/s10434021-10448-6.

ACKNOWLEDGMENTS This study was supported in part by the National Institutes of Health's National Center for Advancing Translational Sciences, Grant No. UL1TR002494. This research also was supported in part by the National Cancer Institute P30 Cancer Center Support Grant, Grant No. CA77598, of Masonic Cancer Center, University of Minnesota. The content is solely the responsibility of the authors and does not necessarily represent the official views of the National Institutes of Health.

DISCLOSURE There are no conflicts of interest.

\section{REFERENCES}

1. Wosik J, Fudim M, Cameron B, et al. Telehealth transformation: COVID-19 and the rise of virtual care. J Am Med Inform Assoc. 2020;27:957-62. https://doi.org/10.1093/jamia/ocaa067.

2. Perrin P, Pierce B, Elliott T. COVID-19 and telemedicine: a revolution in healthcare delivery is at hand. Health Sci Rep. 2020;3:e166. https://doi.org/10.1002/hsr2.166.

3. Bashshur R, Doarn CR, Frenk JM, Kvedar JC, Woolliscroft JO. Telemedicine and the COVID-19 pandemic: lessons for the future. Telemed J E Health. 2020;26:571-3. https://doi.org/10. 1089/tmj.2020.29040.rb.

4. Centers for Medicare \& Medicaid Services. Medicare telemedicine health care provider fact sheet. 2020. Retrieved April 17, 2021 at https://www.cms.gov/newsroom/fact-sheets/medicar e-telemedicine-health-care-provider-fact-sheet.

5. U.S. Department of Health and Human Services Office for Civil Rights. FAQs on telehealth and HIPAA during the COVID-19 nationwide public health emergency. 2020. Retrieved April 17, 2021 at https://www.hhs.gov/sites/default/files/telehealth-faqs-5 08.pdf

6. Serper M, Cubell AW, Deleener ME, et al. Telemedicine in liver disease and beyond: can the COVID-19 crisis lead to action? Hepatology. 2020;72:723-8. https://doi.org/10.1002/hep.31276.

7. Saleem SM, Pasquale LR, Sidoti PA, Tsai JC. Virtual ophthalmology: telemedicine in a COVID-19 era. Am J Ophthalmol. 2020;216:237-42. https://doi.org/10.1016/j.ajo.2020.04.029.

8. Timpel P, Oswald S, Schwarz PEH, Harst L. Mapping the evidence on the effectiveness of telemedicine interventions in diabetes, dyslipidemia, and hypertension: an umbrella review of systematic reviews and meta-analyses. J Med Internet Res. 2020;22:e16791. https://doi.org/10.2196/16791.

9. Cremades M, Ferret G, Parés D, et al. Telemedicine to follow patients in a general surgery department. A randomized controlled trial. Am J Surg. 2020;219:882-7. https://doi.org/10.1016/ j.amjsurg.2020.03.023.

10. Makhni MC, Riew GJ, Sumathipala MG. Telemedicine in orthopaedic surgery: challenges and opportunities. $J$ Bone $J t$ Surg. 2020;102:1109-15. https://doi.org/10.2106/JBJS.20.00452.

11. Forbes RC, Solorzano CC, Concepcion BP. Surgical telemedicine here to stay: more support from a randomized controlled trial on postoperative surgery visits. Am J Surg. 2020;219:880-1. http s://doi.org/10.1016/j.amjsurg.2020.03.033.

12. Steele TA. Chemotherapy-induced immunosuppression and reconstitution of immune function. Leuk Res. 2002;26:411-4. h ttps://doi.org/10.1016/s0145-2126(01)00138-2.

13. Kutikov A, Weinberg DS, Edelman MJ, et al. A war on two fronts: cancer care in the time of COVID-19. Ann Intern Med. 2020;172:756-8. https://doi.org/10.7326/M20-1133.

14. Zhang L, Zhu F, Xie L, et al. Clinical characteristics of COVID19-infected cancer patients: a retrospective case study in three hospitals within Wuhan, China. Ann Oncol. 2020;31:894-901. h ttps://doi.org/10.1016/j.annonc.2020.03.296.

15. Yu J, Ouyang W, Chua MLK, Xie C. SARS-CoV-2 transmission in patients with cancer at a tertiary care hospital in Wuhan, China. JAMA Oncol. 2020;6:1108-10. https://doi.org/10.1001/jamaonc ol.2020.0980.

16. Liang W, Guan W, Chen R, et al. Cancer patients in SARS-CoV2 infection: a nationwide analysis in China. Lancet Oncol. 2020;21:335-7. https://doi.org/10.1016/S1470-2045(20)30096-6.

17. Carlson LE, Angen M, Cullum J, et al. High levels of untreated distress and fatigue in cancer patients. $\mathrm{Br} J$ Cancer. 2004;90:2297-304. https://doi.org/10.1038/sj.bjc.6601887. 
18. Carlson LE, Bultz BD. Cancer distress screening: needs, methods, and models. J Psychosom Res. 2003;55:403-9. https://doi. org/10.1016/s0022-3999(03)00514-2.

19. Zabora J, BrintzenhofeSzoc K, Curbow B, Hooker C, Piantadosi S. The prevalence of psychological distress by cancer site. Psychooncology. 2001;10:19-28. https://doi.org/10.1002/10991611(200101/02)10:1\%3c19::aid-pon501\%3e3.0.co;2-6.

20. Harris PA, Taylor R, Thielke R, Payne J, Gonzalez N, Conde JG. Research electronic data capture (REDCap): a metadata-driven methodology and workflow process for providing translational research informatics support. J Biomed Inform. 2009;42:377-81. https://doi.org/10.1016/j.jbi.2008.08.010.

21. Parmanto B, Lewis AN Jr, Graham KM, Bertolet MH. Development of the telehealth usability questionnaire (TUQ). Int $J$ Telerehabil. 2016;8:3-10. https://doi.org/10.5195/ijt.2016.6196.

22. Mair F, Whitten P. Systematic review of studies of patient satisfaction with telemedicine. BMJ. 2000;320:1517-20. https://doi. org/10.1136/bmj.320.7248.1517.

23. Kitamura C, Zurawel-Balaura L, Wong RK. How effective is video consultation in clinical oncology? A systematic review. Curr Oncol. 2010;17:17-27. https://doi.org/10.3747/co.v17i3. 513.

24. Mair F, Whitten P, May C, Doolittle GC. Patients' perceptions of a telemedicine specialty clinic. $J$ Telemed Telecare. 2000;6:36-40. https://doi.org/10.1258/1357633001933925.

25. Kunkler IH, Rafferty P, Foreman D. A pilot study of tele-oncology in Scotland. J Telemed Telecare. 1998;4:113-9. https://d oi.org/10.1258/1357633981932055.

26. Allen A, Hayes J. Patient satisfaction with teleoncology: a pilot study. Telemed J. 1995;1:41-6. https://doi.org/10.1089/tmj.1.19 95.1.41.

27. Gutkin PM, Prionas ND, Minneci MO, et al. Telemedicine in radiation oncology: is it here to stay? Impacts on patient care and resident education. Int $J$ Radiat Oncol Biol Phys. 2020;108:416-20. https://doi.org/10.1016/j.ijrobp.2020.06.047.
28. Shaverdian N, Gillespie EF, Cha E, Kim SY, Benvengo S, Chino F, Kang JJ, Li Y, Atkinson TM, Lee N, Washington CM, Cahlon O, Gomez DR. Impact of telemedicine on patient satisfaction and perceptions of care quality in radiation oncology. J Natl Compr Canc Netw. 2021;4:1-7. https://doi.org/10.6004/jnccn.2020.7687. Epub ahead of print.

29. Di Lalla V, Patrick H, Siriani-Ayoub N, Kildea J, Hijal T, Alfieri J. Satisfaction among cancer patients undergoing radiotherapy during the COVID-19 pandemic: an institutional experience. Curr Oncol. 2021;28:1507-17. https://doi.org/10.3390/curronc ol28020142.

30. Leon A, Kaltman R, Arem H, et al. Abstract SS2-09: telemedicine usability for cancer care during the COVID-19 pandemic. Cancer Res. 2021;81(4 Suppl):SS2-09. https://doi.org/ 10.1200/JCO.2020.38.29_suppl.265.

31. Bakken S, Grullon-Figueroa L, Izquierdo R, et al. Development, validation, and use of English and Spanish versions of the telemedicine satisfaction and usefulness questionnaire. J Am Med Inform Assoc. 2006;13:660-7. https://doi.org/10.1197/jamia. M2146.

32. Lewis JR. IBM computer usability satisfaction questionnaires: psychometric evaluation and instructions for use. Int J HumComput Int. 1995;7:57-78. https://doi.org/10.1080/ 10447319509526110.

33. Yip MP, Chang AM, Chan J, MacKenzie AE. Development of the Telemedicine Satisfaction Questionnaire to evaluate patient satisfaction with telemedicine: a preliminary study. J Telemed Telecare. 2003;9:46-50. https://doi.org/10.1258/ 135763303321159693.

Publisher's Note Springer Nature remains neutral with regard to jurisdictional claims in published maps and institutional affiliations. 